\title{
Grafting Crown Ether Alkali Host-Guest Complexes at Surfaces by Electrospray Ion Beam Deposition
}

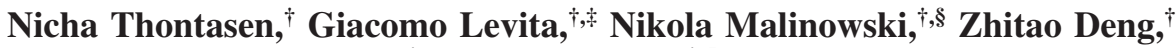 \\ Stephan Rauschenbach, ${ }^{*, \dagger}$ and Klaus Kern ${ }^{\dagger, \pi}$ \\ Max-Planck-Institute for Solid State Research, Nanoscale Science Department, Heisenbergstrasse 1, \\ DE-70569 Stuttgart, Germany, INFM-Democritos Modeling Center for Research in Atomistic Simulation, \\ Trieste, Italy, and Institut de Physique de la Matière Condensée, Ecole Polytechnique Fédérale de Lausanne, \\ CH-1015 Lausanne, Switzerland
}

Received: July 2, 2010; Revised Manuscript Received: August 18, 2010

The functionalization of surfaces with host-guest compounds is promising for many applications, yet often limited by constraints such as the volatility of the functional compound or the lack of binding to the surface. We use electrospray ion beam deposition (ES-IBD) on surfaces in ultrahigh vacuum as a novel approach to modify an atomically defined copper surface with preformed dibenzo-24-crown-8-alkali complexes, in which the central ion $\left(\mathrm{H}^{+}, \mathrm{Na}^{+}\right.$, or $\left.\mathrm{Cs}^{+}\right)$can be exchanged in the electrospray solution. In situ scanning tunneling microscopy maps the single alkali ion complexes as an oval protrusion with a four-lobe submolecular structure immobilized at the surface. Density functional theory calculations confirm that the crown ether is bound to the surface via the central alkali ion within its cavity, indicating that the properties of the molecular complex are retained after deposition.

\section{Introduction}

Host-guest interaction is one of the major fields of research in supramolecular chemistry ${ }^{1}$ and plays a fundamental role in many chemical and biological processes. Macrocycles are particularly attractive as host molecules with tunable functionality. The variety of shapes and chemical compositions characterizing the ring moiety and central ion allows for the fabrication of templates with properties that are tuned by the modification of the independent functional groups at the inner and outer sides of the macrocycle or by the exchange of the central ion. ${ }^{2}$ The functionalization of surfaces with host-guest complexes thus attracts the attention of researchers since it is desirable for many applications, such as molecular recognition, ion sensing, heterogeneous catalysis or molecular switching. ${ }^{3}$

Crown ethers are an important class of macrocycles due to their highly selective ion-affinity, which depends on the cavity size. ${ }^{4}$ Research on the host-guest interactions of crown ethers has been carried out extensively in solution ${ }^{5}$ and in the gas phase ${ }^{6}$ ever since the development of supramolecular chemistry. At the solid-liquid interface, densely packed crown ether layers were imaged with electrochemical STM. ${ }^{7,8}$ However, one part of our study shows that vacuum processing and in situ characterization of crown ethers are hindered by their thermal instability and extreme mobility at the surface (see Supporting Information).

Here we present a novel approach to fabricate surfaces modified with crown ether host-guest-complexes using electrospray ion beam deposition (ES-IBD), a combination of

\footnotetext{
* To whom correspondence should be addressed. E-mail: s.rauschenbach@ fkf.mpg.de.

† Max-Planck-Institute for Solid State Research.

* INFM-Democritos Modeling Center for Research in Atomistic Simulation.

II Ecole Polytechnique Fédérale de Lausanne.

§ Present address: Central Laboratory of Photographic Processes, Bulgarian Academy of Sciences, 1113 Sofia, Acad. G. Bonchev St., B1. 109, Bulgaria.
}

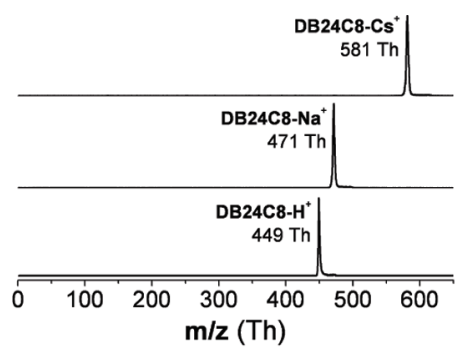

(a)

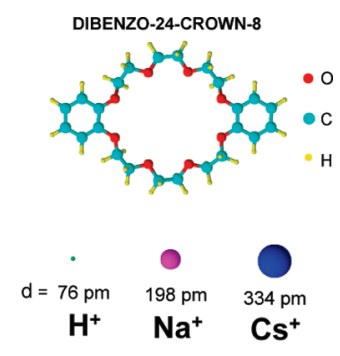

(b)
Figure 1. (a) ESI-TOF mass spectra of DB24C8- Cs ${ }^{+}, \mathrm{DB} 24 \mathrm{C} 8-\mathrm{Na}^{+}$, and DB24C8- $\mathrm{H}^{+}$ion beams for IBD. (b) Structural model of DB24C8 and of the incorporated ions (diameter given).

electrospray ionization $(\mathrm{ESI})^{9}$ and soft-landing deposition. ${ }^{10-13}$ As a model system, the complex of a large crown ether, dibenzo-24-crown-8 (DB24C8, see Figure 1b), and positive ions $\left(\mathrm{H}^{+}, \mathrm{Na}^{+}\right.$, and $\left.\mathrm{Cs}^{+}\right)$are investigated on an atomically defined metal surface $(\mathrm{Cu}(100))$ by in situ scanning tunneling microscopy (STM) in ultrahigh vacuum (UHV). By simply choosing the salt added to the electrospray solution, the central cation of the deposited complex can be selected. The surfaces are coated with ultimate control in UHV using mass selected beams of solely the desired compound. STM reveals the molecular substructure of the deposited complexes in which the central ion can be identified. Density function theory (DFT) calculations show that the ion retains its charge upon adsorption at the metal surface, hence its chemical state and thus its functionality is preserved to a great extent. Moreover, the crown ether complex is more strongly bound than the crown ether alone. ES-IBD is applicable to many soluble compounds, both volatile and nonvolatile, which opens manyfold possibilities for surface functionalization with molecular host-guest complexes. 


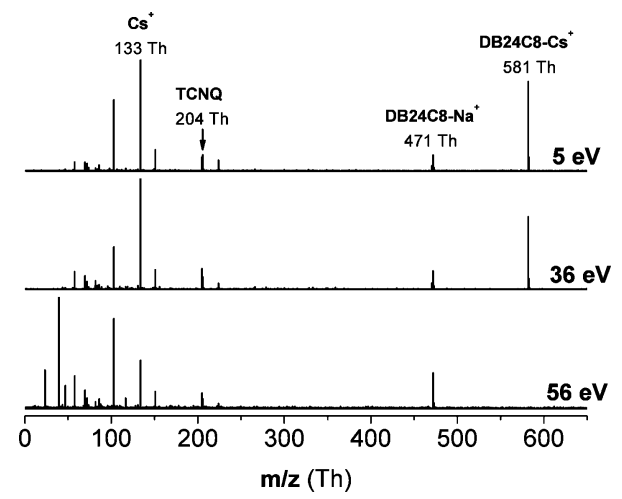

Figure 2. Ex situ MALDI-TOF mass spectra of DB24C8- $\mathrm{Cs}^{+}$after IBD in high vacuum at beam energies of 5,36, and $56 \mathrm{eV}$. The intact complex DB24C8- $\mathrm{Cs}^{+}$is detected at $581 \mathrm{Th}$ for 5 and $36 \mathrm{eV}$ deposition energy. At $56 \mathrm{eV}$, no complex is found. TCNQ (204 u) is used as a matrix. The peaks at 133 and $471 \mathrm{Th}$ can be identified as $\mathrm{Cs}^{+}$and DB24C8- $\mathrm{Na}^{+}$, respectively.

\section{Results and Discussion}

Electrospray Mass Spectrometry. Ion beams of three different DB24C8-cation complexes were prepared, characterized, and deposited on a $\mathrm{Cu}(100)$ surface in UHV (see Methods Section). The incorporation of cations $\mathrm{Cs}^{+}, \mathrm{Na}^{+}$, and $\mathrm{H}^{+}$in the complex was achieved by adding $\mathrm{CsI}, \mathrm{NaCl}$, and formic acid to a solution of DB24C8. Mass spectra shown in Figure 1a were acquired by a built-in time-of-flight (TOF) mass spectrometer prior to deposition to ensure the purity of the beam. From top down, mass spectra of DB24C8- $\mathrm{Cs}^{+}$, DB24C8- $\mathrm{Na}^{+}$, and DB24C8- $\mathrm{H}^{+}$complexes are displayed. The corresponding massto-charge-ratios $(\mathrm{m} / z)^{14}$ of the three peaks are $581 \mathrm{Th}, 471 \mathrm{Th}$, and $449 \mathrm{Th}$, which correspond to singly charged complexes of DB24C8 with one $\mathrm{Cs}^{+}, \mathrm{Na}^{+}$, or $\mathrm{H}^{+}$ion as respective charge carrier. ${ }^{15}$ The absence of any other peaks of significant intensity in the mass spectra indicates the high purity of the beams. In particular, no evidence for the protonated neutral complexes, that is DB24C8-Cs-H ${ }^{+}$or DB24C8-Na-H ${ }^{+}$, was found.

Ex situ MALDI Mass Spectrometry. To confirm soft landing of the crown ether complexes upon IBD, ex situ matrixassisted laser desorption ionization mass spectrometry (MALDITOF) was employed on samples prepared in high vacuum. The $\mathrm{Cs}^{+}$complex was chosen for this experiment because it can only originate from ion beam deposition. In contrast, the $\mathrm{Na}^{+}$ complex can be formed as well by DB24C8 with $\mathrm{Na}^{+}$ contamination that always exists in ex situ characterization. DB24C8- $\mathrm{Cs}^{+}$ion beams were deposited at various incidence energies on a surface covered by 7, 7, 8, 8-tetracyanoquinodimethane (TCNQ), which acts as a matrix. ${ }^{16}$

Figure 2 shows MALDI-TOF mass spectra obtained at beam energies of 5, 36, and $56 \mathrm{eV}$. At $581 \mathrm{Th}$, the peak corresponding to the deposited DB24C8- $\mathrm{Cs}^{+}$complex disappears when the beam energy is as high as $56 \mathrm{eV}$. Besides this, many other peaks occur particularly in the low $\mathrm{m} / z$-range. The peak at $204 \mathrm{Th}$, appearing in all three mass spectra, corresponds to singly charged TCNQ, which was used as a matrix. At $102 \mathrm{Th}$, a fragment of TCNQ can be observed. The peak at $133 \mathrm{Th}$ corresponds to $\mathrm{Cs}^{+}$whereas the peak at $471 \mathrm{Th}$ can be attributed to the DB24C8- $\mathrm{Na}^{+}$complex. These ions are observed for all beam energies.

The intact DB24C8- $\mathrm{Cs}^{+}$complex has been found at the surface if the deposition energy was below $36 \mathrm{eV}$, while its fragments, including $\mathrm{Cs}^{+}$, were found for high incidence energies. From the ESI-TOF mass spectra in Figure 1 and the MALDI measurements it can be concluded that the disintegra- tion of the complex at high incidence energies is caused by crash landing, since there is no fragmentation in the gas phase before deposition. These energy values are in agreement with previous studies in molecular ion beam deposition. ${ }^{12,17}$ DB24C8$\mathrm{Na}^{+}$is generally observed as well in the MALDI mass spectra, despite the fact that it has not been deposited. This observation can be explained by the ex situ preparation and handling of the matrix samples, which inevitably causes sodium contamination. During the ionization in the plasma plume of the MALDI-MS some crown ethers can exchange their central cation.

Since a low deposition energy of $5 \mathrm{eV}$ was set for all the IBD experiments in UHV, soft landing deposition can safely be assumed. The regular structures observed in the STM measurements confirm that intact molecular complexes have been deposited to the surface (see Figure 3).

Scanning Tunneling Microscopy and DFT Calculations. After deposition of a desired coverage of the complex in UHV, the $\mathrm{Cu}(100)$ sample was transferred in situ to the STM. Figure 3 shows STM topographs ${ }^{18}$ obtained after cooling the sample to $43 \mathrm{~K}$, whereas at room temperature the molecules were highly mobile and a well-defined molecular structure could not be observed. At low temperature, the general feature observed for all three complexes is the presence of an oval-shaped structure of an apparent height of $(1.8 \pm 0.2) \AA$ with a lateral size of $12-15 \AA$ along the short axis, and $16-21 \AA$ along the long axis (see Figure $3 \mathrm{~g}$ ). ${ }^{19}$ For the alkali ion complexes of DB24C8 with $\mathrm{Cs}^{+}$and $\mathrm{Na}^{+}$, molecular substructure consisting of four lobes is visible. Because the crown ethers are not shapepersistent, small variations in shape can occur, which is reflected in the observed distributions for the length of axes of approximately $\pm 1.5 \AA$.

In the DB24C8-Cs ${ }^{+}$complexes (Figure 3d), an additional bright feature at the center can be observed (red arrow in Figure $3 \mathrm{~g}$ ). Also, one of the lobes on the short axis is typically found to be significantly brighter than the others. For $\mathrm{Na}^{+}$, both lobes on the short axis are equally bright and their relative positions are very close. The structure appears to be more elongated than the $\mathrm{Cs}^{+}$complex and an additional feature in the center cannot be observed.

For DB24C8- $\mathrm{H}^{+}$complexes, a single bright lobe without any substructure is observed. Since the DB24C8- $\mathrm{H}^{+}$ion beam was occasionally contaminated by a small amount of DB24C8$\mathrm{Na}^{+}$complexes, some STM topographs show 4-lobe features of the $\mathrm{Na}^{+}$complex together with the single lobe structures that correspond to the $\mathrm{H}^{+}$complex, proving that the absence of an internal structure in the DB24C8- $\mathrm{H}^{+}$complexes is not due to poor STM-tip condition.

Evaporation of DB24C8 alone and the coevaporation of Cs atoms did not produce results comparable to IBD. At low coverage, the molecules show extremely high mobility. In contrast to the DB24C8- $\mathrm{H}^{+}$complex, even at $43 \mathrm{~K}$ no immobilized crown ethers could be observed by STM. At higher coverage or after Cs atom dosing, unordered fractal structures are generally observed at the surface. Neither a coating of isolated molecules nor complexes could be produced by evaporation. The fact that the results were not even reproducible indicates that by standard vacuum deposition no well-defined layer of crown ether complexes can be formed at the surface (see Supporting Information).

To interpret the observed structures after ion beam deposition, theoretical modeling of the DB24C8-cation complexes, adsorbed at the surface, was carried out from DFT-based calculations. The obtained adsorption geometries for the DB24C8- $\mathrm{Cs}^{+},-\mathrm{Na}^{+}$, and $-\mathrm{H}^{+}$complexes are illustrated in Figure 4. For the most 

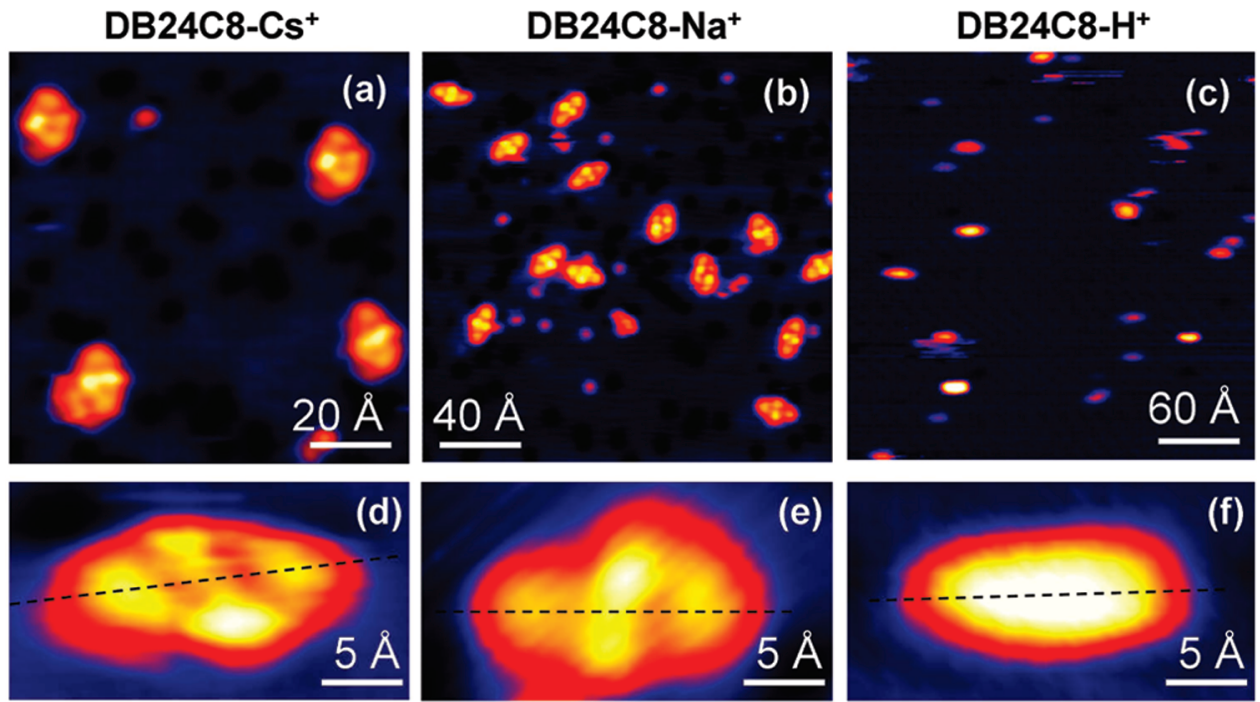

(g)

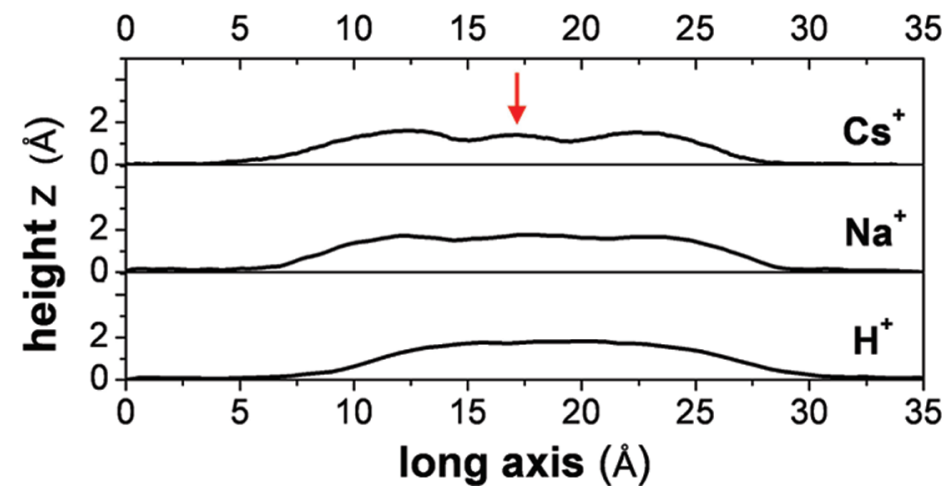

Figure 3. Overview and magnified single complex STM topographies of DB24C8-cations complexes on Cu(100) prepared by IBD. (a,d) DB24C8$\mathrm{Cs}^{+},(\mathrm{b}, \mathrm{e}) \mathrm{DB} 24 \mathrm{C} 8-\mathrm{Na}^{+}$, and $(\mathrm{c}, \mathrm{f}) \mathrm{DB} 24 \mathrm{C} 8-\mathrm{H}^{+}$. (g) Line profiles along the long axis displayed at an equal lateral and vertical scale.
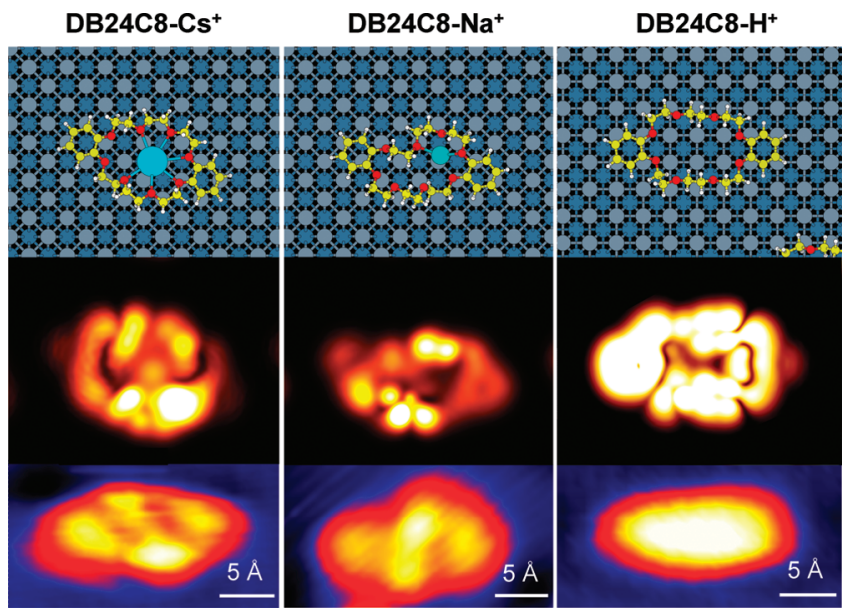

Figure 4. (top row) Geometry of the $\mathrm{DB} 24 \mathrm{C} 8$ complex at the $\mathrm{Cu}(100)$ surface. DB24C8- $\mathrm{Cs}^{+},-\mathrm{Na}^{+}$, and $-\mathrm{H}^{+}$from left to right. (middle row) Simulated STM images according to the above structures. (bottom row) Magnified STM topographs of single complexes.

stable structures of the adsorbed DB24C8 complexes, STM images were computed by integrating the density of states between the bias potential $(-1.3 \mathrm{eV})$ and the Fermi energy, following the Tersoff-Hamann approach. ${ }^{20} \mathrm{~A}$ comparison of the resulting images at a tip height of about $1.2 \AA$ above the molecule is shown in Figure 4.

The images reflect the DB24C8 geometrical structure with the benzene rings located at the edges of the longer axis. Because of their low height above the surface, they are imaged as fainter areas in contrast to the lifted ether chains, which in turn give rise to the bright spots near the center of the structures. The $\mathrm{Cs}^{+}$complex exhibits a centrosymmetric disposition of the bright lobes with one brighter side corresponding to a higher position of the ether chain. The $\mathrm{Na}^{+}$complex displays an S-shaped pattern with the central lobes having comparable intensity. In the sodium complex, the resulting structure is strongly asymmetric. The folding of one side of the chain toward the center of the ring leads to an S-shaped central cavity. The corresponding STM image appears more elongated than the one for the cesium complex, which shows a more compact, oval-shaped conformation. The simulated images also differ in the middle of the cavity region where the ion is located. While $\mathrm{Na}^{+}$is virtually not imaged, the larger $\mathrm{Cs}^{+}$is mapped as a light spot in the center of the simulated image.

On the basis of the good agreement between the experimental and theoretical STM images, the calculations strongly support the experimental evidence of complex binding between the alkali ions and DB24C8 via coordination around the central cavity. The absence of self-assembled domains suggests that the complex, which has been deposited as an ion, is partially positively charged at the surface as well. As a consequence, the system is characterized by repulsive molecule-molecule interactions and chemisorption on the metal surface. This observation agrees well with the findings on Cs-organometallic networks on $\mathrm{Cu}(100){ }^{21}$ The adsorption energies and the limited charge transfer at the interface reveal that chemisorption is relatively weak and is taking place only between the alkali ion 
and the surface. However, the overall adsorption process is affected also by the weaker interactions between the organic parts of the complex and the copper.

Physisorption of pure $\mathrm{DB} 24 \mathrm{C} 8$ on $\mathrm{Cu}(100)$ is mostly mediated by the interaction of the benzene rings with the metal surface. The well-known inadequacies in the DFT description of van der Waals interactions prevent a detailed analysis of the adsorption mechanism, which is however estimated as being exothermic for about $0.2-0.3 \mathrm{eV}$. The DFT modeling shows that the coordination of an alkali ion into the adsorbed DB24C8 results in a much more favored adsorption on $\mathrm{Cu}(100)$. The adsorption energies for the DB24C8- $\mathrm{Na}^{+}$and $-\mathrm{Cs}^{+}$complexes were calculated as 1.17 and $1.72 \mathrm{eV}$, respectively, which is in the characteristic range for chemisorption binding.

Depending on the size of the central ion and its interactions with the host molecule and the surface, the location of the ion inside the ring cavity and the morphology of the complex vary. In the STM topographies the size of the central cavity is found to scale with the size of the ions, that is, $\mathrm{Cs}^{+}>\mathrm{Na}^{+}$; for $\mathrm{H}^{+}$in particular the substructure cannot be observed.

The most stable adsorption geometry for the DB24C8- $\mathrm{Na}^{+}$ complex suggests that the $\mathrm{Na}^{+}$ion is coordinated to four oxygen atoms near one of the two benzene rings. For the DB24C8$\mathrm{Cs}^{+}$complex, the alkali center is coordinated to six oxygen atoms. The proton in the DB24C8- $\mathrm{H}^{+}$complex was found to hardly affect the adsorption geometry and the consequent STM imaging. Nevertheless, its presence in the complex at the surface is indicated by the different behavior of the DB24C8- $\mathrm{H}^{+}$(Figure 3c) complex compared to evaporated DB24C8 (see Supporting Information, Figure S1a).

For a large molecule, many conformational states of similar adsorption energies are expected. In the experiment, we find structures with small differences in shape compared to those most commonly observed. DFT calculations show that the potential energy surface for the adsorbed systems is relatively flat, as witnessed by the presence of numerous local minima within less than $0.1 \mathrm{eV}$. This gives rise to many possible orientations on the surface and small deviations in shape. Minima on the potential energy landscape differ by small displacements of the metal center or parts of the organic ring with respect to the $\mathrm{Cu}(100)$ surface. While the binding occurs via chemisorption at the alkali ions, the rest of the complex is less strongly interacting with the copper and consequently retains some degrees of freedom. Moreover, the comparison of the binding energies of uncoordinated $(0.3 \mathrm{eV})$ and alkalicoordinated DB24C8 (1.17 or $1.72 \mathrm{eV}$ ) explains the experimental evidence of a significant difference in mobility.

\section{Conclusion}

The in situ ion beam deposition experiments presented here demonstrate a new route for the highly controlled surface modification with large molecules that are otherwise difficult to handle. Crown ether complexes, which are large and fragile functional molecular systems, were successfully deposited on a metal surface by means of ES-IBD. Conventional sublimation processing failed to produce analogous results. In particular, the fabrication of crown ether complexes at the surface by coevaporation of the constituents proved to be erratic, indicating that no well-defined surface modification is achieved.

On the other hand, ES-IBD is able to perform the desired complex by mixing its constituents in solution under ambient conditions and deposit it onto the surface in UHV in a welldefined way. In addition, parameters such as the deposition energy or the fluence are controlled, both of which can be measured directly through the deposition current with high precision.

Analysis by STM at $43 \mathrm{~K}$ with submolecular resolution allowed to identify the type and conformation of the complexes, which was confirmed by DFT calculations. It is found that the crown ether is adsorbed flat on the $\mathrm{Cu}(100)$ substrate with the cation placed within the cavity. DFT calculations show that the deposition as preformed complex enables a strong binding to the surface. Moreover, the charge of the molecular complex is retained after deposition.

ES-IBD proves to be a highly versatile method for the deposition of organic material in UHV. To deposit three different complexes, the same methodology can be applied while the only difference in the procedure is the preparation of the solution. ESI in general works for a large variety of nonvolatile compounds. It can thus be expected that all of those can be deposited with ES-IBD in UHV. Nonvolatile substances such as many polymers, proteins, or metal-organic complexes that so far were not available for vacuum deposition before can now be used to make a surface coating. Electrospray ion beam deposition, in particular in combination with STM analysis, thus opens up manifold perspectives for surface functionalization and molecular nanotechnology.

\section{Methods Section}

Electrospray Ion Beam Deposition. For deposition in UHV and subsequent in situ characterization, an ES-IBD source was constructed, which extends the setup presented before. ${ }^{17,22}$ The creation of the gas phase ions takes place at ambient pressure by pneumatically assisted ESI at a flow rate of $1 \mathrm{~mL} / \mathrm{h}$. Dibenzo24-crown-8 (DB24C8) (Aldrich 253197) was dissolved in dichloromethane and mixed with solutions of $\mathrm{EtOH}$ and water (50\% each) of $\mathrm{NaCl}$ (Sigma-Aldrich 204439), CsI (Harshaw Chemical G-2153) and formic acid (FA, Fluka 94318) resulting in solutions for electrospray containing the crown ether and the counterion at the same concentration of $10^{-3} \mathrm{M}$. If not given otherwise, all chemicals used were analytical grade.

The ions are transferred into vacuum and guided through six differentially pumped stages to reach a pressure below $10^{-10} \mathrm{mbar}$ at the sample position. The setup integrates different ion optics, such as a skimmer, rf-quadrupoles, or electrostatic lenses, that allow the formation and collimation of an ion beam, mass selection, focusing, and beam deflection. Throughout the whole system the ion current can be monitored on several relevant electrodes using electrometers (Keithley 617). The kinetic energy of the ion beam can be characterized by a retarding grid detector. Chemical characterization is performed by an integrated linear TOF-MS with a mass resolution of $m / \Delta m \approx 300$. This instrument allows for the unambiguous identification of the complexes after careful calibration. Sample holders in the fourth and sixth pumping stage are specifically designed to adjust the deposition energy and monitor the deposition current. The samples in the sixth pumping stage at UHV can be transferred in situ to the STM, while the sample holder in the fourth pumping stage contains the samples that are used for ex situ characterization.

At the substrate position in UHV, mass selected ion currents of 5-10 pA were reached. The incidence energy for the deposition was adjusted to $5 \mathrm{eV}$ for all the experiments in UHV in order to achieve soft landing. For each deposition, the fluence ${ }^{23}$ was estimated by the integration of the beam current. For the deposition of DB24C8-Cs ${ }^{+}$and $-\mathrm{Na}^{+}$complexes, the fluence has been approximately $50 \mathrm{pAh}$, whereas it had been reduced to $25 \mathrm{pAh}$ in the case of $\mathrm{H}^{+}$due to the significantly 
lower initial electrospray current. Thereby $50 \mathrm{pAh}$ corresponds to a coverage of $10 \%$ of a monolayer (ML) of DB24C8-alkali ion complexes on the substrate surface of $20 \mathrm{~mm}^{2}$ while 25 pAh corresponds to $5 \% \mathrm{ML}$ coverage of $\mathrm{DB} 24 \mathrm{C} 8-\mathrm{H}^{+}$. According to that, a deposition usually lasted several hours, which leads to frequent contamination of the $\mathrm{Cu}(100)$ surface by oxygen or CO, imaged as depression of $50 \mathrm{pm}$ in the STM topographs (see Figure $3 a-c$ ).

Chemical Surface Characterization. Ex situ MALDI-TOF (Bruker Reflex IV, Bruker Daltonik GmbH, Bremen, Germany) was used to detect the deposited crown ether complexes to confirm the chemical integrity after IBD. The mass spectrometer was operated in positive-ion mode with the laser power adjusted to $20 \%$ of the full scale. In the forth pumping stage of the IBD source at $10^{-7}$ mbar, ion beams of DB24C8- $\mathrm{Cs}^{+}$complexes were deposited on $\mathrm{Si}$ samples $(7 \mathrm{~mm} \times 7 \mathrm{~mm})$ coated with the matrix 7, 7, 8, 8-tetracyanoquinodimethane (TCNQ) (Alfa Aesar $\mathrm{GmbH}$, Karlsruhe, Germany). Substrate preparation was carried out by sublimation of TCNQ molecules onto the Si samples at $200{ }^{\circ} \mathrm{C}$ for a few minutes under ambient conditions. ${ }^{16}$ The incidence energy was varied for different samples by applying voltages to the substrate.

Scanning Tunneling Microscopy. A single crystalline $\mathrm{Cu}(100)$ sample (MaTeck GmbH, Juelich, Germany) was used as the substrate for all UHV depositions. Surface preparation by repeated $\mathrm{Ar}^{+}$sputtering at $1 \mathrm{kV}(15 \mathrm{~min})$ followed by annealing at $800 \mathrm{~K}(5-15 \mathrm{~min})$ resulted in a clean substrate surface with extended terraces, which was confirmed by STM before deposition.

After the deposition, the surface was characterized by means of a variable temperature STM (Omicron Nanotechnology $\mathrm{GmbH}$, Taunusstein, Germany) performed in UHV at room temperature and at about $43 \mathrm{~K}$. The STM was cooled by liquid helium through a flow-cryostat. Bias voltages in the range of -0.9 to $-1.5 \mathrm{~V}$ and a tunneling current of $25-80 \mathrm{pA}$ were typical values applied to all measurements.

DFT Calculations. DFT modelization of the adsorbed DB24C8 complexes on the $\mathrm{Cu}(100)$ surface was carried out by means of the QuantumESPRESSO package, ${ }^{24}$ which makes use of plane-wave expansion of the system wave function in periodically repeated supercells. We used a $21.6 \times 18.0 \times 18.0$ $\AA^{3}$ tetragonal cell; the $\mathrm{Cu}(100)$ surface was modeled by just two $\mathrm{Cu}$ layers due to the large lateral extension of the cell, necessary to avoid interactions between adsorbates in adjacent periodic images. The positions of the copper atoms of the lowest layer were kept frozen at the equilibrium bulk geometry during the optimization processes. Calculations were performed with the Perdew-Burke-Ernzerhof exchange-correlation functional, ${ }^{25}$ using Vanderbilt-type ultrasoft potentials ${ }^{26}$ with a kinetic energy cutoff of $40 \mathrm{Ry}$; this led to a nearest-neighbor $\mathrm{Cu}-\mathrm{Cu}$ distance of $2.55 \AA$, which is in good agreement with the experimental value. For cesium, we used a norm-conserving Troullier-Martins pseudopotential ${ }^{27}$ due to the unavailability of ultrasoft potentials; convergence tests proved that the $40 \mathrm{Ry}$ cutoff represents a satisfactory compromise between energetic/ geometric accuracy and sensible computational time usage. The $k$-points sampling was limited to the Gamma point.

The adsorption energies of the DB24C8-alkali complexes were determined by the deposition of the complex with a $\mathrm{Cl}^{-}$counterion to avoid computation of an overall charged supercell. STM images were simulated by means of the specific tool in the QuantumESPRESSO package, based on the Tersoff-Hamann approach. ${ }^{20}$ All images were produced using the XCrySDen program. ${ }^{28}$
Acknowledgment. The authors would like to thank Dr. S. Stepanow, Dr. M. Lingenfelder, Dr. H. Nuss, Dr. A. Langner, and Dr. Thomas Weitz for their support and for many fruitful discussions. We acknowledge the instrumental support by Professor Bill.

Supporting Information Available: Description of the material included. This material is available free of charge via the Internet at http://pubs.acs.org.

\section{References and Notes}

(1) (a) Cragg, P. J. A Practical Guide to Supramolecular Chemistry; Wiley: West Sussex, 2005. (b) Steed, J. W.; Turner, D. R.; Wallace, K. J. Core Concepts in Supramolecular Chemistry and Nanochemistry; Wiley: West Sussex, 2007; pp 29-106. (c) Lehn, J.-M. Supramolecular Chemistry; VCH: Weinheim, 1995; pp 4-5. 908.

(2) Leininger, S.; Olenyuk, B.; Stang, P. J. Chem. Rev. 2000, 100, 853-

(3) (a) Bradshaw, J. S.; Izatt, R. M. Acc. Chem. Res. 1997, 30, 338345. (b) Gokel, G. W.; Leevy, W. M.; Weber, M. E. Chem. Rev. 2004, 104, 2723-2750. (c) Ruben, M.; Payer, D.; Landa, A.; Comisso, A.; Gattinoni, C.; Lin, N.; Collin, J.-P.; Sauvage, J.-P.; De Vita, A.; Kern, K. J. Am. Chem. Soc. 2006, 128, 15644-15651. (d) Payer, D.; Rauschenbach, S.; Konuma, M.; Virojanadara, C.; Starke, U.; Dietrich, C.; Collin, J.; Sauvage, J. P.; Lin, N.; Kern, K. J. Am. Chem. Soc. 2007, 129, 15662 15667.

(4) Pedersen, C. J. J. Am. Chem. Soc. 1967, 89, 7017-7036.

(5) Izatt, R. M.; Pawlak, K.; Bradshaw, J. S.; Bruening, R. L. Chem. Rev. 1991, 91, 1721-2085.

(6) Maleknia, S.; Brodbelt, J. J. Am. Chem. Soc. 1992, 114, 42954298.

(7) Ohira, A.; Sakata, M.; Hirayama, C.; Kunitake, M. Org. Biomol. Chem. 2003, 1, 251-253.

(8) Xu, L.-P.; Wan, L.-J. J. Phys. Chem. B 2006, 110, 3185-3188.

(9) Fenn, J. B.; Mann, M.; Meng, C. K.; Wong, S. F.; Whitehouse, C. M. Science 1989, 246, 64-71.

(10) Franchetti, V.; Solka, B. H.; Baitinger, W. E.; Amy, J. W.; Cooks, R. G. Int. J. Mass Spectrom. Ion Processes 1977, 23, 29-35.

(11) Ouyang, Z.; Takats, Z.; Blake, T. A.; Gologan, B.; Guymon, A. J.; Wiseman, J. M.; Oliver, J. C.; Davisson, V. J.; Cooks, R. G. Science 2003, $301,1351-1354$.

(12) Laskin, J.; Wang, P.; Hadjar, O. Phys. Chem. Chem. Phys. 2008, 10, 1079-1090.

(13) Laskin, J.; Yang, Z.; K., C. I. J. Am. Chem. Soc. 2008, 130, 32183230

(14) Given in units of Thomson (Th); $1 \mathrm{Th}=1 \mathrm{u} / \mathrm{e}^{29}$

(15) Franski, R. Rapid Commun. Mass Spectrom. 2009, 23, 3488-3491.

(16) (a) Przybilla, L.; Brand, J. D.; Yoshimura, K.; Rader, H. J.; Mullen, K. Anal. Chem. 2000, 72, 4591-4597. (b) Rouhanipour, A.; Roy, M.; Feng, X. L.; Räder, H. J.; Müllen, K. Angew. Chem., Int. Ed. 2009, 48, 46024604.

(17) Rauschenbach, S.; Vogelgesang, R.; Malinowski, N.; Gerlach, J. W.; Benyoucef, M.; Costantini, G.; Deng, Z. T.; Thontasen, N.; Kern, K. Acs Nano 2009, 3, 2901-2910.

(18) Images were processed by WSXM. ${ }^{30}$

(19) Long and short axes are defined by connecting the maxima of the submolecular features in the topography.

(20) (a) Tersoff, J.; Hamann, D. R. Phys. Rev. Lett. 1983, 50, 19982001. (b) Tersoff, J.; Hamann, D. R. Phys. Rev. B 1985, 31, 805-813.

(21) Stepanow, S.; Ohmann, R.; Leroy, F.; Lin, N.; Strunskus, T.; Woell, C.; Kern, K. ACS Nano 2010, 4, 1813-1820.

(22) Rauschenbach, S.; Stadler, F. L.; Lunedei, E.; Malinowski, N.; Koltsov, S.; Costantini, G.; Kern, K. Small 2006, 2, 540-547.

(23) In this paper, the fluence is defined as the deposited charge. It is given here in units of picoampere-hour (pAh).

(24) Giannozzi, P.; et al. J. Phys.: Condens. Matter 2009, 21, 395502.

(25) Perdew, J.; Burke, M.; Ernzerhof, K. Phys. Rev. Lett. 1996, 77, 3865

(26) Vanderbilt, D. Phys. Rev. B 1990, 41, 8412.

(27) Troullier, N.; Martins, J. L. Phys. Rev. B 1991, 43, 1993.

(28) Kokalj, A. Comput. Mater. Sci. 2003, 28, 155.

(29) Cooks, R. G.; Rockwood, A. L. Rapid Commun. Mass Spectrom. 1991, $5,93$.

(30) Horcas, I.; Fernandez, R.; Gomez-Rodriguez, J. M.; Colchero, J.; Gomez-Herrero, J.; Baro, A. M. Rev. Sci. Instrum. 2007, 78, 013705.

JP106123Q 